Pirineos. Revista de Ecología de Montaña

Vol. 174

Jaca, Enero-Diciembre, 2019, e047

ISSN-1: 0373-2568

https://doi.org/10.3989/pirineos.2019.174007

\title{
EL MICROREFUGIO DE UCHUCAY: UN RELICTO DE BOSQUE INTERANDINO CON UNA IMPORTANTE RIQUEZA ARBÓREA EN EL SUR DEL ECUADOR
}

\section{The Uchucay microrefugium: an Interandean forest relict with an important arboreal richness in Southern Ecuador}

\author{
Danilo Minga ${ }^{1}$, Paula Cordero ${ }^{2}$, Mario Donoso-Correa ${ }^{2-3}$, Kabir Montesinos $^{3}$, \\ Mayra Jimenez ${ }^{1}$, Berea Antaki ${ }^{4}$, Fausto Sarmiento ${ }^{4 *}$ \\ ${ }^{1}$ Herbario HA, Universidad del Azuay. Cuenca, Ecuador. \\ ${ }^{2}$ Facultad de Ingeniería, Universidad Católica de Cuenca, Ecuador. \\ ${ }^{3}$ Proyecto VLIR Migración. Universidad de Cuenca, Ecuador. \\ ${ }^{4}$ Colaboratorio de Montología Neotropical, Departamento de Geografía, Universidad de Georgia. Athens, GA 30602 USA.
}

\author{
Identificador ORCID de los autores y e-mail \\ Danilo Minga: http://orcid.org/0000-0003-2398-2740. Email: dminga@uazuay.edu.ec \\ Paula Cordero: http://orcid.org/0000-0002-4510-3316. Email: paulac70@hotmail.com \\ Mario Donoso-Correa: http://orcid.org/0000-0002-0505-4118. Email: mdonosoc@ucacue.edu.ec \\ Kabir Montesinos: http://orcid.org/0000-0002-9954-6948. Email: kabir.montesinos@ucuenca.edu.ec \\ Mayra Jimenez: http://orcid.org/0000-0003-3339-060X. Email: mjimenez@uazuay.edu.ec \\ Berea Antaki: http://orcid.org/0000-0002-9473-4413.Email: berea.antaki25@uga.edu \\ Fausto Sarmiento: http://orcid.org/0000-0003-0501-6020. Email: fsarmien@uga.edu \\ *Autor corresponsal
}

Recibido: 29-04-2019. Aceptado: 21-08-2019. Fecha de publicación on-line: 19/09/2019

Citation / Cómo citar este artículo: Minga, D., Cordero, P., Donoso-Correa, M., Montesinos, K., Jimenez, M., Antaki, B., Sarmiento, F. (2019). El microrefugio de Uchucay: un relicto de bosque interandino con una importante riqueza arbórea en el sur del Ecuador. Pirineos, 174, e047. https://doi.org/10.3989/pirineos.2019.174007

RESUMEN: La Reserva Comunitaria de Uchucay mantiene un importante relicto de bosque nativo interandino, parte de la Mancomunidad El Collay de Vegetación y Bosque Protector en el austro ecuatoriano, que ha sido conservado por su relevancia en la provisión del caudal hídrico, pero del que se conocía poco en cuanto a su biodiversidad. Mediante transectos de $50 \times 2 \mathrm{~m}$, se estudió la riqueza y composición florística de especies leñosas con un diámetro a la altura del pecho (DAP) igual o superior a $2,5 \mathrm{~cm}$. Se registraron 57 especies arbóreas, pertenecientes a 47 géneros y 32 familias, con una riqueza que oscila entre 12 y 18 especies por hectárea y una diversidad alfa promedio de 2,5 en el índice de Shannon. La composición florística está caracterizada por la predominancia de especies como: Weinmannia fagaroides, Columellia oblonga y Clusia flaviflora; en tanto que la densidad muestra un promedio de 67 individuos por hectárea, con un área basal que alcanza los $0,77 \mathrm{~mm}^{2}$ por hectárea $(77 \mathrm{~m} / \mathrm{ha})$; estos resultados indican que son bosques antiguos con una elevada diversidad arbórea. Además, constituyen un microrefugio importante para 
especies amenazadas como Prumnopitys montana, Podocarpus oleifolius, Oreopanax avicenniifolius, Miconia hexamera y Gynoxys dielsiana. La investigación incluyó un estudio sobre la percepción del cambio ambiental y de qué manera se afectan los pobladores al perder especies emblemáticas del bosque nativo, como el Podocarpus sp.

PALABRAS CLAVE: Microrefugio, biodiversidad arbórea, especie emblemática, bosque andino nativo, Azuay.

\begin{abstract}
The Uchucay Community Reserve harbors a native interandean forest relict that has been conserved because of its role in water capture and supply, within the larger El Collay Commonwealth Protected Forest and Vegetation of southeastern Ecuador. However, little is known about its tree biodiversity. Using $50 \times 2 \mathrm{~m}$ transects randomly located, we studied the composition of woody plants with a diameter at breast height (DBA) equal or larger than $2.5 \mathrm{~cm}$. We registered 57 tree species that belong to 47 genera and 32 families, with a richness between 12 and 18 species/ha and alpha diversity of 2.5 Shannon Index. The floristic composition is dominated by Weinmannia fagaroides, Columellia oblonga and Clusia flaviflora; while the density shows an average if 67 individuals per hectare, with basal area near-ing $0.77 \mathrm{~mm}^{2} /$ ha or roughly $77 \mathrm{~m}^{2} /$ ha. These results show that they are old forests with high tree diver-sity. Also, that it is a microrefugium for endangered species, such as Prumnopitys montana, Podocarpus oleifolius, Oreopanax avicenniifolius, Miconia hexamera and Gynoxys dielsiana. The study included a survey on local environmental perception and the way in which they are affected with the loss of native forest flagship species, like Podocarpus sp.
\end{abstract}

KEYWORDS: Microrefugium, tree biodiversity, flagship species, Andean forest, Azuay.

\section{Introducción}

Los bosques tropicales montanos de neblina (BTMN) constituyen un elemento importante de los Andes ecuatorianos, donde la mayor diversidad florística parece concentrarse en esta región, con aproximadamente el 64\% de especies del Ecuador (Jorgensen \& León-Yánez, 1999). Mulligan (2011) señala que, para el año 2009, en los Andes tropicales (Venezuela, Colombia, Ecuador, Perú y Bolivia) la remanencia de estos bosques es de 605.317 $\mathrm{km}^{2}$, de los cuales $63.323 \mathrm{~km}^{2}(10,4 \%)$ se encuentran en Ecuador. En el mismo estudio se señala que el área deforestada de estos bosques es de $560.499 \mathrm{~km}^{2}$ en 2009. En el Ecuador está la franja de vegetación de alta montaña que se extiende desde los 2500 hasta los 3800 metros sobre el nivel del mar (m.s.n.m.) y se caracteriza por poseer altas concentraciones de humedad durante la mayor parte del año, aunque no necesariamente haya mucha lluvia, debido a la precipitación horizontal que engolfa estos bosques con nubosidad diaria. Los árboles pueden alcanzar bastante altura de fuste (Hofstede et al., 1998) dependiendo del sustrato, el relieve y la plasticidad fenotípica de cada especie. En los países andinos tropicales se identifican tres regiones continentales, a saber, sierra alta, selva baja y costa marina. Los bosques montanos son considerados simplemente como elementos transicionales entre la altura (las zonas de la cordillera alta sobre los 3.800 m.s.n.m.) y la bajura (las zonas de la llanura aluvial bajo los 600 m.s.n.m.). Nosotros insistimos en considerarlos como una región en sí misma, la de los flancos cordilleranos, el píe de monte o los contrafuertes andinos, o usar los descriptores locales: bosques nubosos, nuboselva, selva de neblina, ceja de montaña, ceja de selva o yungas.

Estos bosques de neblina o nuboselvas (Sarmiento, 1988), además de ser reservorios de biodiversidad y tener un valor cultural por los beneficios que las comunidades pueden obtener de ellos, son importantes proveedores de servicios ecosistémicos principalmente vinculados al agua (Bruijnzeel et al., 2011), a la regulación climática regional (Jarvis \& Mulligan, 2011) y a la captura y almacenamiento de carbono. Las nuboselvas se encuentran catalogadas como ecosistemas frágiles y estratégicos por estar situados en zonas de recarga de cuencas hidrográficas que proveen agua que beneficia a millones de personas en Colombia, Bolivia, Ecuador y Perú (Cuesta et al., 2009). Condit et al. (2000) sugieren que estos ecosistemas de montaña se consideran paisajes forestales de pocas especies, aunque en algunas áreas se encuentran rodales puros de una misma especie, como por ejemplo en los bosques altoandinos monoespecíficos de Polylepis spp.

Sin embargo, desde tiempos inmemoriales estos ecosistemas forestales han estado sujetos a perturbaciones que han provocado la fragmentación del bosque, la pérdida de especies y la degradación de sus servicios ecosistémicos, llevando consigo cambios importantes en su composición, estructura y funcionamiento (McGarical \& Cushman, 2002). Es así que en la actualidad se han convertido en mosaicos compuestos por potreros y tierras de labor abandonadas, fragmentos de bosque secundario, escasos relictos de bosque remanente, matorrales con vegetación de subpáramo, extensiones de cultivos y plantaciones forestales, principalmente eucalipto (Eucalyptus globulus) y pino de Monterrey (Pinus radiata) (VelascoLinares \& Vargas, 2007). Sin embargo, debido a la presencia de relictos entre los páramos circundantes y los bosques remanentes que sobreviven a la intensa deforestación de las épocas colonial (siglos XVI-XVIII) y republicana (siglos XIX y XX), éstas pequeñas áreas remanentes mantienen los valores de patrimonio biocultural que se debieran proteger para los escenarios futuros del 
cambio climático (Sarmiento et al., 2018), convirtiéndolas en de-facto microrefugios bioculturales (Figura 1). En este estudio intentamos caracterizar el relicto y ubicarlo dentro de la percepción local respecto de la necesidad de conservar la cobertura del bosque y, en especial, especies emblemáticas como puede ser el guabisay ( $P_{o-}$ docarpus sp).

Los BTMN del Ecuador del flanco andino-amazónico son continuos y muy húmedos. Por el otro lado, en las estribaciones occidentales, los BTMN son extensos y continuos en Colombia y en el norte de Ecuador; pero más hacia el sur, la franja del BTMN en los repliegues cordilleranos es menos húmedo y más estrecho, terminando en el departamento de Túmbez, Perú, cerca de la frontera con Ecuador (Sarmiento, 2001; Pennington et al., 2004). Así los bosques montanos bajos del sur de Ecuador/norte de Perú constituyen la extensión más sureña de los BTMN de la ecoregión chocoandina. Sin embargo, cerca de su límite austral, Valencia et al., (2000) enfatizan la diversidad y el endemismo presente en las estribaciones tanto orientales como occidentales, debido principalmente a la depresión de Huancabamba en el Paso de Porculla, constituyendo la altitud más baja de toda la cordillera andina, lo que ha favorecido una mezcla de flora andina, amazónica y tumbesina, afectadas por un intercambio cultural muy antiguo entre la sierra, la selva y la costa, haciendo de las especies existentes un reflejo de la dinámica natura-cultura en los paisajes tropandinos (Sarmiento, 2016). De esta manera, muchas especies pueden ser endémicas en pequeñas áreas y más aún en la región austral donde son menos conocidas. Esto hace que las cifras record de endemismo se presenten en estos paisajes colinados pedemontinos así como en las estribaciones y flancos cordilleranos. Por esta razón, pensamos que es prioritario adoptar una nueva estrategia de conservación biocultural (Sarmiento \& Viteri, 2015), incluyendo la conservación de

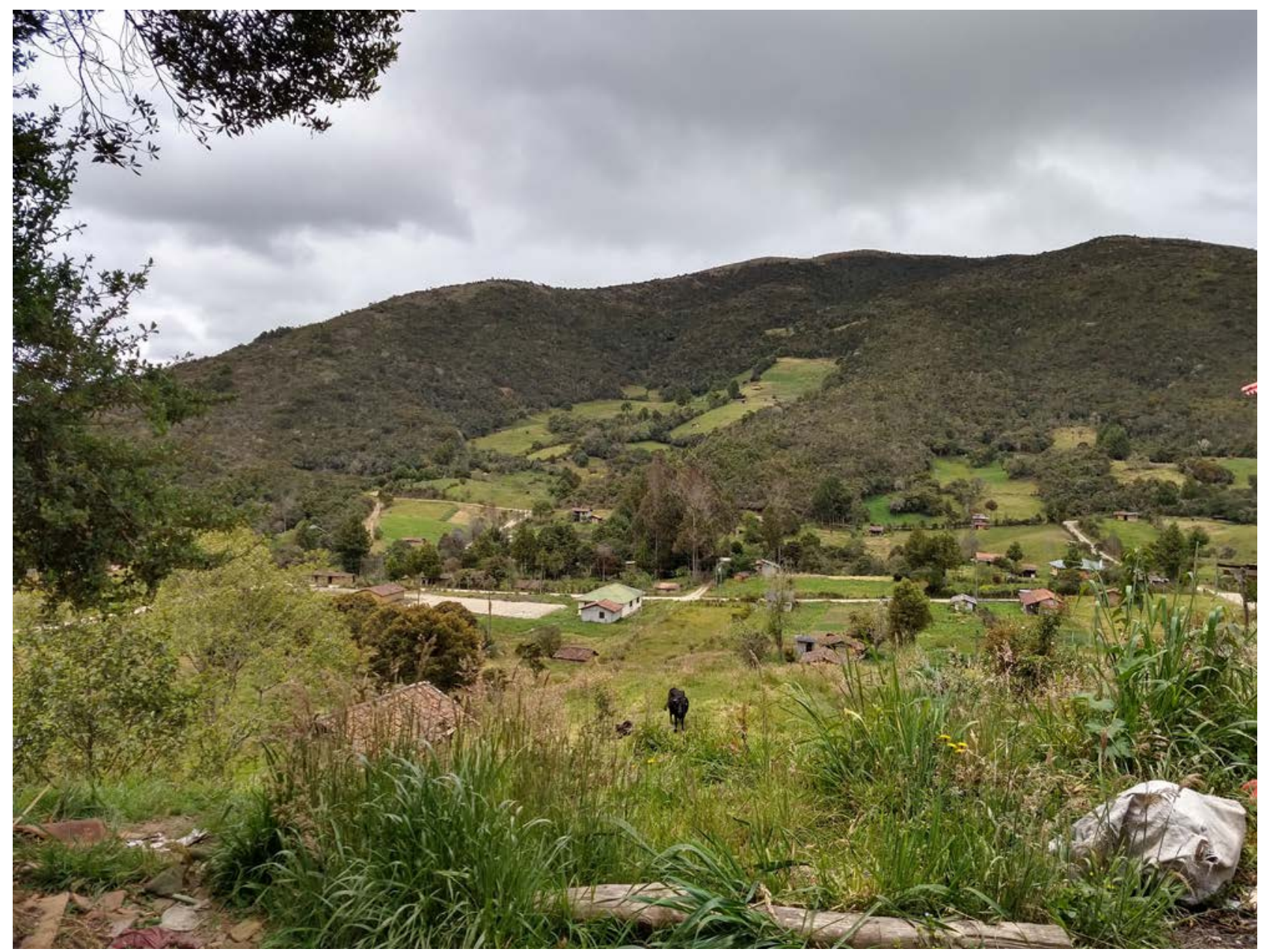

Figura 1: Panorámica del bosque de la Reserva Comunitaria Uchucay, perteneciente a la mancomunidad El Collay, en la provincia del Azuay, en el austro ecuatoriano. Aquí se observa la dinámica natura/cultura que hace de estos microrefugios bioculturales algo importante en la planificación del desarrollo sustentable en montañas. Fotografía: Mario Donoso.

Figure 1: Panoramic view of the Uchucay Community Reserve of the El Collay Commonwealth, in the Azuay province of Southern Ecuador. The nature/culture dynamics observed hereby makes these biocultural microrefugia something important in the planning of sustainable mountain development. Photograph: Mario Donoso. 
microrefugios a lo largo de estos paisajes socio-ecológicos productivos donde la biodiversidad persiste a pesar de la influencia humana, o gracias a ella.

\subsection{Problemática y objetivos de investigación}

Bajo estos antecedentes surge la necesidad de investigar los relictos de BTMN en la región e identificar sus servicios ecosistémicos como posibles microrefugios de especies amenazadas y micrositios reguladores del caudal hídrico, para contribuir con información que facilite la elaboración y ejecución de planes enfocados a la conservación de estos microrefugios de flora arbórea andina. Se ha propuesto que los riscos aislados y los acantilados de altura, que son literalmente intocados por la influencia humana, deberían ser prioritarios para conservación (Ledo et al., 2009). Sin embargo, hemos observado que el gran despliegue de biodiversidad se da en el límite de los paisajes humanizados y los relictos de bosque remanente, en donde la matriz del paisaje cultural ha facilitado la supervivencia de plantas y animales. Estos microrefugios son ahora considerados vitales para el mantenimiento del estilo de vida tradicional de las comunidades de montaña. El programa internacional de la Iniciativa Satoyama de la Universidad de las Naciones Unidas (UNU) lo considera de alta prioridad para la sustentabilidad humana debido a que son los mejores ejemplos de paisajes socioecológicos terrestres y marinos productivos. La mayoría de las evaluaciones realizadas ecológicamente apuntan a prioridades de investigación que incluyan paisajes fusionados, manufacturados ancestralmente, dentro de la malla para la preservación de la diversidad del patrimonio biocultural (Mathez-Stiefel et al., 2017), no solamente la protección de extensos territorios de áreas protegidas como parques nacionales y reservas equivalentes, sino también en los microrefugios y corredores de conectividad biocultural.

\section{Metodología}

\section{1. Área de estudio}

El estudio se efectuó en la comunidad de Uchucay, perteneciente a la parroquia Luis Cordero Vega del Cantón Gualaceo (Figura 2). El área corresponde a una zona

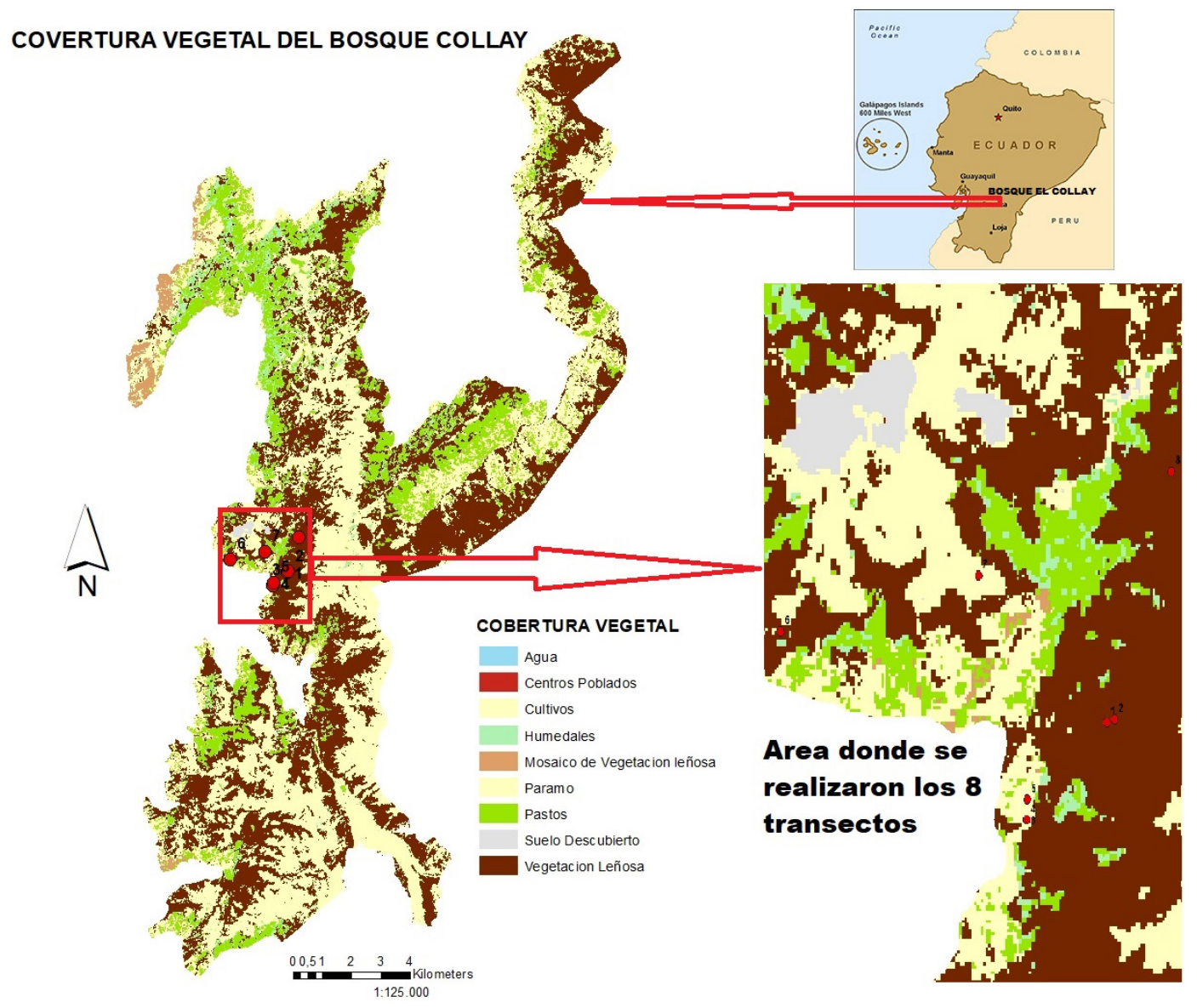

Figura 2: Mapa de ubicación del bosque Collay en el austro ecuatoriano, mostrando en el recuadro la distribución de transectos y áreas de muestreo en la reserva comunitaria de Uchucay, cercana a Gualaceo. Elaboración: Mario Donoso.

Figure 2: Location map of the El Collay forest in southern Ecuador showing an insert box with the distribution of transects and sampling areas in the Uchucay Community Reserve, near Gualaceo. Adaptation: Mario Donoso. 
montañosa que comprende un pequeño valle, rodeado por colinas de mediana altitud (entre 2800 m.s.n.m. a 3200 m.s.n.m.) localizada geográficamente a $2^{\circ} 54 ' 17^{\prime \prime}$ latitud Sur y a $78^{\circ} 43^{\prime} 05^{\prime}$ ' longitud Oeste (http://visitagualaceo. travel/destinations/uchucay/). El plano del valle ha sido deforestado para la producción agrícola y ganadera de subsistencia; así, la mayor superficie de esta zona está ocupada por pastizales introducidos con especies como: raygrass (Lolium perenne L.), pasto azul u holco (Holcus lanatus L.), cluva (Anthoxanthum odoratum L.) y kikuyo (Pennisetum clandestinum Hochst. ex Chiov.), que se complementan con cultivos de melloco, avena, cebada, habas y maíz con fréjol.

La vegetación nativa corresponde a la formación vegetal de bosque siempre verde montano alto (Sierra et al., 1999). La mayor parte de la vegetación nativa se encuentra en las laderas que rodean al valle en altitudes comprendidas entre 3.100 m.s.n.m. y 3.400 m.s.n.m. (Figura 3) Esta zona se encuentra cubierta por un bosque con un dosel entre 8 y $12 \mathrm{~m}$ de altura con predominancia de especies como: Weinmannia fagaroides Kunth., Clusia flaviflora Engl., y Clethra fimbriata Kunth. En el subdosel se encuentran arbolitos y arbustos como Viburnum triphyllum Benth., Oreopanax avicennifolius Dechne. \&

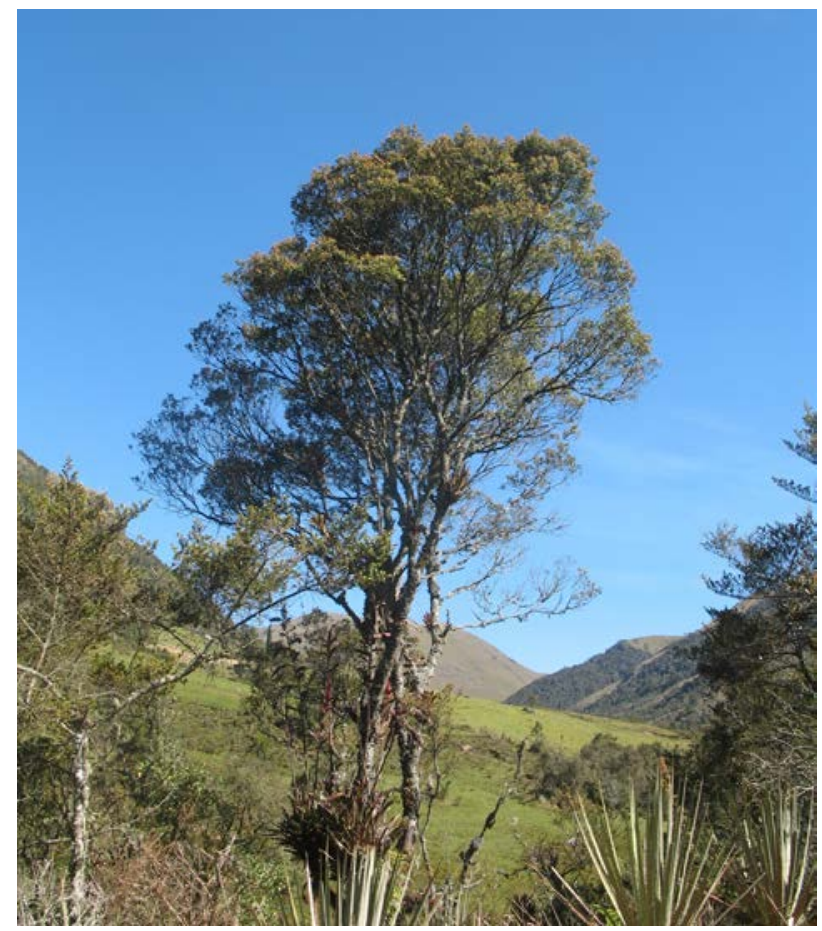

Figura 3: Una vista del paisaje de montaña con los remanentes de bosque andino en una ladera y en primer plano el Sarar

(Weinmannia faragoides), y el resto con la matriz antrópica del páramo y los pastizales en ruta a Uchucay. Fotografía: Paula Cordero.

Figure 3: A view of the mountainscape with Andean forests remnants on a slope, and the 'Sarar' (Weinmannia

faragaloides) in the foreground, and the rest with the anthropic matrix of the páramo grassland in the route to Uchucay. Photograph: Paula Cordero.
Planch., Gynoxys cuicochensis Cuatrec., Miconia aspergillaris Naudin., entre otras. En la zona alta, por encima de los 3400 m.s.n.m., se encuentra la zona de páramo arbustivo con predominancia de especies de los géneros Calamagrostis, Monticalia, Disterigma, Gaultheria, Brachyotum, Baccharis, Gynoxys y Macleania (Figura 4).

\subsection{Métodos de campo}

Con la finalidad de documentar la diversidad de la flora arbórea del sector, se seleccionaron las áreas boscosas mejor conservadas y se emplazaron transectos de 50x2m (Gentry, 1982); los transectos se trazaron con el lado más largo de forma paralela a la pendiente máxima por exigencia topográfica convencional. En total se efectuaron ocho transectos y en cada uno se midieron todos los tallos de leñosas con un diámetro a la altura de pecho (DAP) igual o superior a $2,5 \mathrm{~cm}$. También se estimó su altura con la ayuda de un clinómetro Suunto y se colectó una muestra botánica de hojas, flores, frutos y en algunos casos corteza, para su posterior identificación y herborización en el Herbario HA de la Universidad del Azuay. Cada punto de muestreo fue georeferenciado de acuerdo al Sistema Geodésico Mundial 1984 (WGS84), en la proyección Universal Transversa de Mercator (UTM), zona 17. Se procedió además a realizar 30 entrevistas y a registrar en fotografía y en video las visitas, las especies, y los vecinos que aceptaron ser fotografiados y entrevistados, siguiendo las preguntas de un formulario de encuestas aprobado (IBR-UGA) para la conducción convencional de estudios a sujetos humanos (Figura 5) de conocimiento previo informado.

\subsection{Fase de Herbario}

En total se colectaron 78 muestras botánicas, mismas que fueron prensadas, secadas e identificadas en el Herbario de la Universidad del Azuay (HA) y convertidas en especímenes catalogados de la colección Herbario Azuay (2019). Para la determinación taxonómica se utilizaron las monografías publicadas en la colección de Flora of Ecuador, volúmenes número 4 (Columelliaceae: Fagerström, 1975), 8 (Compositae-Liabeae: Robinson, 1978), 24 (Loranthaceae: Kuijt, 1986), 27 (Cyatheaceae: Tyron, 1986), 40 (Chloranthaceae: Todzia, 1990), 43 (Symplocaceae: Ståhl, 1991), 45 (Clethraceae: Gustafsson, 1992), 54 (Ericaceae: Luteyn, 1996), 56 (Rosaceae: Romoleroux, 1996), 59 (Siparunaceae: Renner \& Hausner, 1996), 61 (Cunoniaceae: Harling, 1999), 62 (Rubiaceae-Coussareeae: Taylor, 1999), 69 (Proteaceae: Bonifaz \& Cornejo, 2002), 77 (Compositae-Heliantheae: Robinson, 2007a) y 83 (Compositae-Eupatorieae: Robinson, 2007b). También se consultó el Catálogo de plantas Vasculares del Ecuador (Jorgensen y León- Yánez, 1999), además de bases de datos y herbarios virtuales: JSTOR (2019), Field Museum Herbarium (2019), el herbario virtual del New York Botanical Garden (2019) y TROPICOS (2019). 


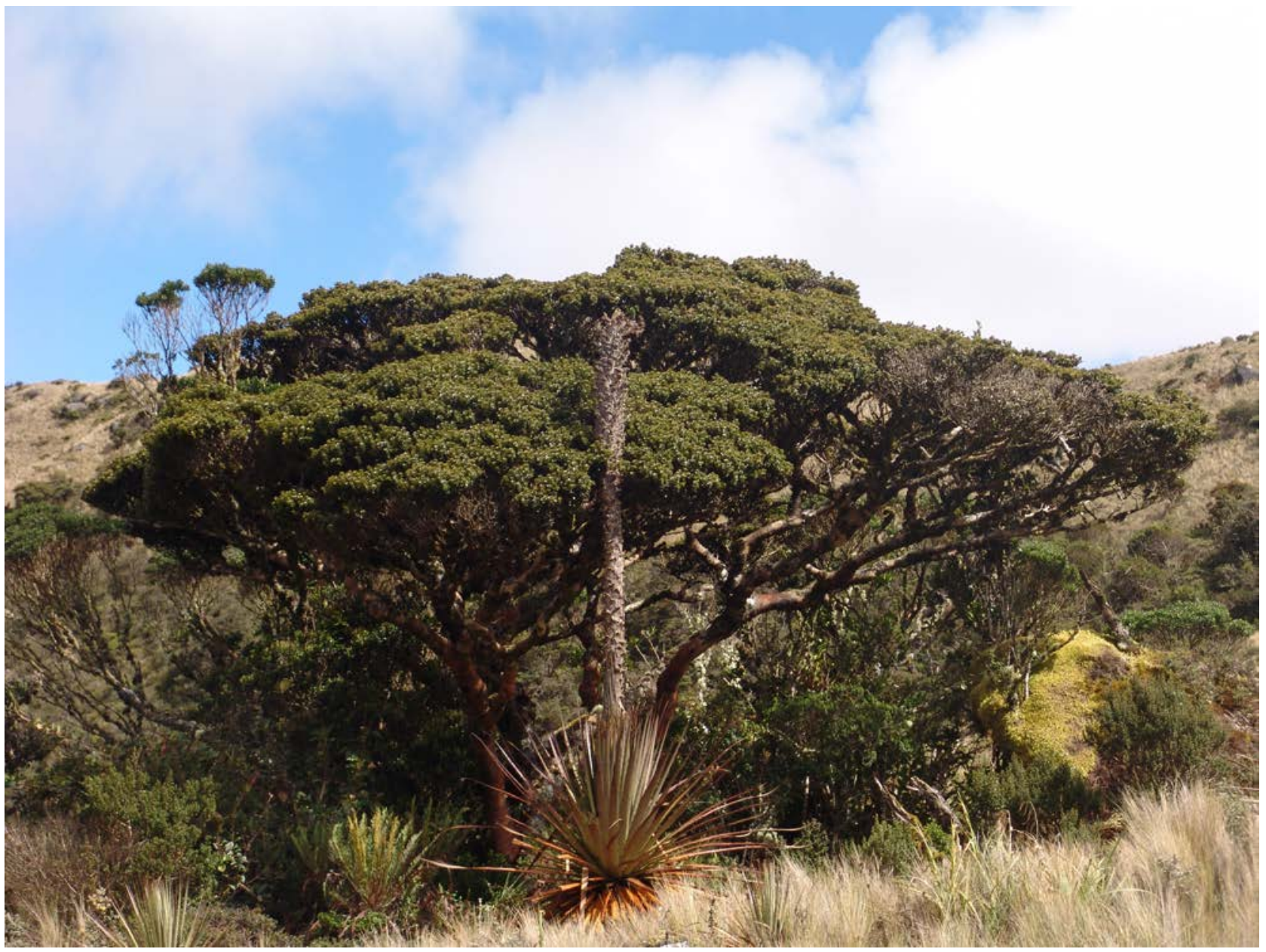

Figura 4: Vista panorámica de los microrefugios formados por los árboles nativos remanentes como Guagra manzana (Hesperomeles ferruginea) en medio de arbustos y pajonal del páramo (Calamagrostis sp, Festuca sp) modificado por la deforestación, la presión de herbivoría y el continuo uso del territorio. Fotografía: Paula Cordero.

Figure 4: Panoramic view of the microrefugium formed by native remnant trees, such as 'Guagra manzana'(Hesperomeles ferruginea), surrounded by shrubs and páramo grassland (Calamagrostis $\mathrm{sp}$, Festuca $\mathrm{sp}$ ) in an area modified by deforestation, the herbivorous' pressure and the continuous use of the territory. Photograph: Paula Cordero.

Para la clasificación taxonómica a nivel de familia se utilizó el sistema APG IV (APG IV, 2016). Finalmente, las colecciones botánicas fueron etiquetadas de acuerdo a las normas estándares del manejo de herbarios y un ejemplar de cada espécimen fértil fue depositado en el herbario HA de la Universidad del Azuay. Así mismo la información sistematizada se encuentra en la base de datos del herbario, parte de esta información se encuentra en línea.

\subsection{Análisis de datos}

En el análisis de datos se calculó la densidad, expresada como densidad relativa (DR) que es proporcional al número de individuos de esa especie en la parcela o transecto con respecto al número total de individuos en el área muestreada ( $0.01 \mathrm{ha}$ ) y se expresa como: $\mathrm{DR}=\mathrm{N}^{\circ} \mathrm{de}$ individuos de una especie / $\mathrm{N}^{\circ}$ Total de individuos en la parcela $X$ 100: el área basal $(A B)$ que se calcula a partir del DAP mediante la expresión siguiente: $\mathrm{AB}=\Omega\left(\mathrm{D}^{2} / 4\right)$, donde: $\Omega$ es 3.141592 y D² es el diámetro. La dominancia, que se refiere al grado de importancia que una o más especies tienen en la estructura y funcionamiento del sistema, se calcula de acuerdo al área basal total y el área basal que la especie ocupa en el área muestreada. El Índice de Valor de Importancia (IVI) es un parámetro que mide el valor de las especies en base a tres parámetros principales: dominancia (ya sea en forma de cobertura o área basal), densidad y frecuencia; por lo tanto, $I V I=$ densidad relativa + frecuencia + dominancia relativa (Mostacedo \& Fredericksen, 2000).

También se calcularon los índices de diversidad de Shannon-Weaver $\left(\mathrm{H}^{\prime}\right)$ y de Simpson (D1) para el efecto se emplearon las fórmulas siguientes (Hill, 1973):

$$
\text { Shannon-Weaver } \quad H=-\sum_{i=1}^{s} p_{i} \log _{e} p_{i}
$$




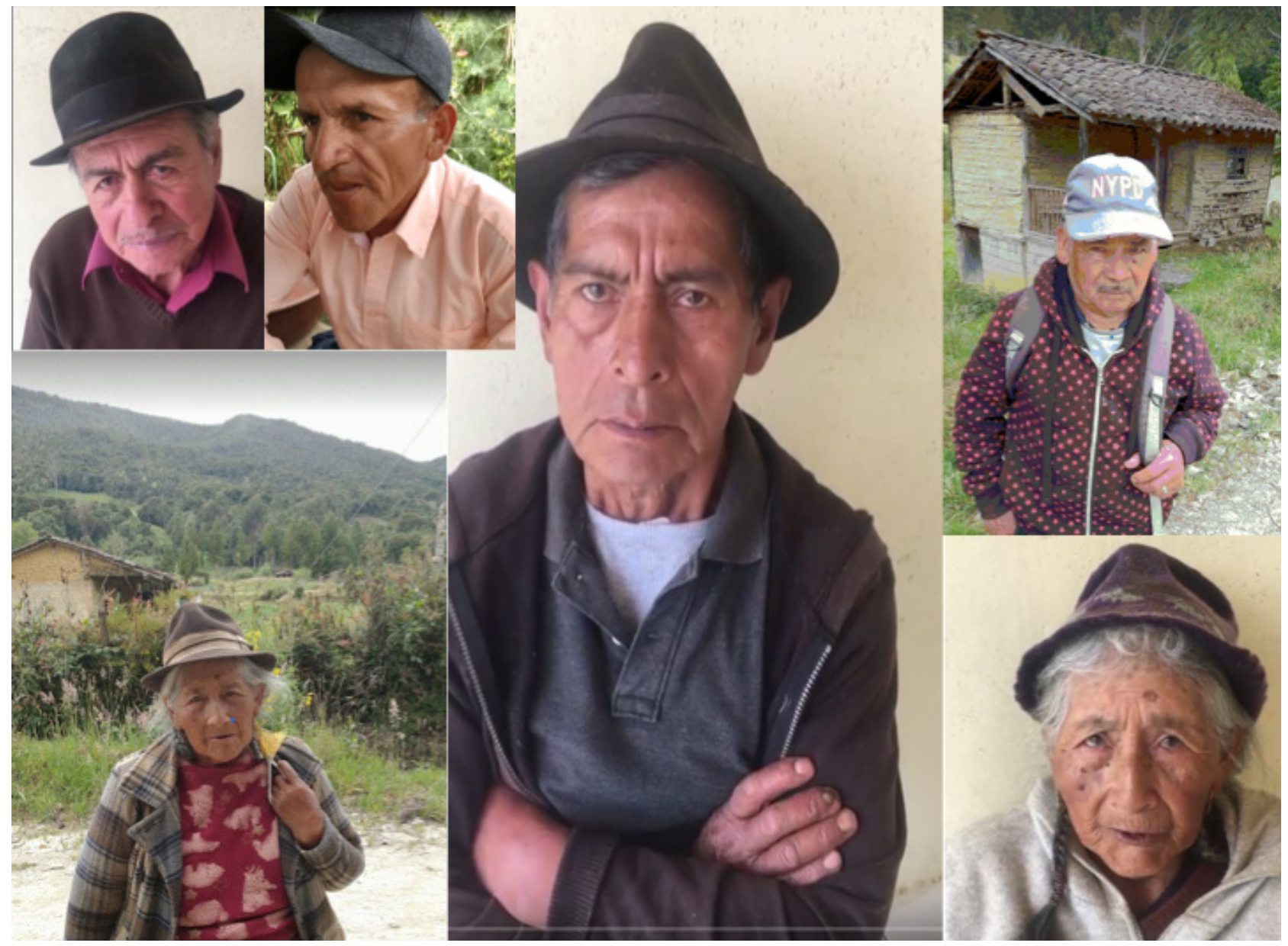

Figura 5: Una pequeña muestra de los comuneros que accedieron voluntariamente con información sobre su percepción sobre el cambio climático y la deforestación de especies emblemáticas como el Romerillo o el Chaquiro (Podocarpus oleifolus, Prumnopitys montana) como ejemplo de las personas entrevistadas en la Reserva Comunitaria de Uchucay. Fotografías: Mario Donoso.

Figure 5: A small sample of the community members who agreed voluntarily to inform us about their environmental perception about climate change and deforestation of flagship species, such as 'Romerillo' and 'Chaquiro'(Podocarpus oleifolus, Prumnopitys montana) as examples of the people interviewed in the Uchucay Community Reserve. Photographs: Mario Donoso.

$$
\text { Simpson } \quad D 1=1-\sum_{i=1}^{s} p_{i}^{2}
$$

Donde $p_{i}$ es la proporción de especies $i, \mathrm{y} S$ es el número de especies de modo que $\sum_{i=1}^{s} p_{i}=1$, y $e$ es la base del logaritmo y el índice de equitatividad, calculado a partir del índice de Shannon-Weaver de acuerdo con la fórmula siguiente:

$$
J=\frac{H}{\ln (S)}
$$

En donde la variable "H" es el índice de ShannonWiener y la variable "S" es la riqueza de especies detectadas durante el muestreo (Carmona-Galindo \& Carmona, 2013).

Adicionalmente, atendiendo a que las muestras al azar pueden representar probabilidades sesgadas por la domi- nancia o por la rareza de las especies en la zona estudiada, se procedió a establecer la probabilidad de ocurrencia del indicador Chao-1 basado en la abundancia de "doubletons", de acuerdo al uso mundial que se ha dado a los estimadores no paramétricos de Chao-1 de abundancia y Chao-2 de incidencia, es decir presencia/ausencia, calculado en línea en http://viceroy.eeb.uconn.edu/estimates.

\subsection{Estado de conservación}

Para conocer el estado de conservación de las especies endémicas registradas en el área se utilizó el Libro Rojo de las plantas endémicas del Ecuador (León-Yánez et al., 2011) y se complementó con la información de encuesta en terreno a moradores y vecinos del área de la Reserva Comunitaria de Uchucay en la mancomunidad El Collay. 


\section{Resultados}

\subsection{Diversidad}

Se registraron 57 especies pertenecientes a 47 géneros y 32 familias (Anexo 1). El análisis de diversidad alfa (Tabla 1), indica que son bosques medianamente diversos, con una riqueza de especies que oscila entre 12 y 18 y una diversidad promedio de 2,5 en el índice de Shannon. De acuerdo con los índices de diversidad y equitatividad, los transectos 5 y 4 son los más diversos, mientras que, de acuerdo con el estimador Chao-1, el sitio del transecto 6 sería el de mayor riqueza de especies.

\subsection{Estructura y composición florística}

La densidad promedio registrada en los ocho transectos, indica que son bosques densos con un dosel tupido de coronas imbricadas que alcanzan un promedio de 67 tallos por polígono (Tabla 2), en tanto que el área basal muestra un promedio de $0,77 \mathrm{~m}^{2}$ por polígono, lo cual significa 77 $\mathrm{m}^{2}$ por ha, un valor que corresponde a bosques antiguos.

La composición está caracterizada por la predominancia de especies como Weinmannia fagaroides, Columellia oblonga y Clusia flaviflora, que son árboles dominantes que conforman el dosel superior del bosque; otras especies importantes son: Rugea sp, Oreopanax sp, Viburnum triphyllum y Myrsine coriacea que son especies que, aunque abundantes, se encuentran bajo el dosel. En la tabla 3 se muestran las 20 especies con mayor Índice de Valor de Importancia, en la misma se nota la predominancia de Weinmannia fagaroides (sarar), que está presente en 7 de los 8 transectos y que muestra una considerable densidad en cada transecto. Clusia flaviflora (duco), muestra similares características fitosociológicas. Columellia oblonga (pispilo) aunque importante de acuerdo con el IVI, es poco frecuente y poco abundante, pero sus individuos son de gran tamaño. Especies como Oreopanax sp (pumamaqui), Viburnum triphyllum (rañas), Myrsine andina (yubar), son especies de tamaño medio que son frecuentes en el área y muestran una moderada abundancia.

\subsection{Endemismo}

Si bien se registran únicamente tres especies endémicas a saber: Oreopanax avicenniifolius, Miconia hexámera y Gynoxys dielsiana; se han encontrado especies de árboles nativos importantes y poco frecuentes en la región

Tabla 1: Comparación de la riqueza y diversidad alfa en 8 transectos de bosque de Uchucay.

Table 1: Comparison of Species Richness and Alpha Diversity in eight transects of the Uchucay forest.

\begin{tabular}{|c|c|c|c|c|c|}
\hline $\mathbf{N}^{\circ}$ de transecto & $\mathbf{N}^{\circ}$ de especies & Índice de Simpson & Índice de Shannon & Equitatividad & Chao-1 \\
\hline T 1 & 19 & 0,8802 & 2,512 & 0,853 & 20,25 \\
\hline T 2 & 15 & 0,8866 & 2,424 & 0,8952 & 18,75 \\
\hline T 3 & 13 & 0,8889 & 2,361 & 0,9205 & 16 \\
\hline T 4 & 17 & 0,9055 & 2,557 & 0,9026 & 20,33 \\
\hline T 5 & 19 & 0,93 & 2,768 & 0,9402 & 19,6 \\
\hline T 6 & 18 & 0,8929 & 2,514 & 0,8698 & 21,33 \\
\hline T 7 & 16 & 0,8713 & 2,409 & 0,869 & 16,6 \\
\hline T 8 & 19 & 0,8835 & 2,473 & 0,8398 & 24 \\
\hline
\end{tabular}

Tabla 2: Características topográficas, densidad y área basal en 8 transectos de 0,01 ha del bosque de Uchucay.

Table 2: Topographic characteristics, Density and Basal area in eight transects of 0.01ha in the Uchucay forest.

\begin{tabular}{|c|c|c|c|c|}
\hline $\mathbf{N}^{\circ}$ de Transecto & Pendiente (grados) & Relieve & $\mathrm{N}^{\circ}$ de individuos & Área Basal m² \\
\hline Transecto 1 & 16 & ladera & 66 & 0,87 \\
\hline Transecto 2 & 8 & valle & 42 & 1,77 \\
\hline Transecto 3 & 12 & valle & 39 & 1,29 \\
\hline Transecto 4 & 14 & valle & 63 & 0,36 \\
\hline Transecto 5 & 15 & ladera & 80 & 0,82 \\
\hline Transecto 6 & 27 & cresta & 91 & 0,3 \\
\hline Transecto 7 & 18 & valle & 63 & 0,48 \\
\hline Transecto 8 & 30 & cresta & 92 & 0,26 \\
\hline TOTAL & & & 536 & 6,14 \\
\hline PROMEDIO & & & 67 & $\mathbf{0 , 7 7}$ \\
\hline
\end{tabular}


Tabla 3: Frecuencia, Densidad e Índice de Valor de Importancia de las especies leñosas en 8 transectos de 0,01 ha del bosque de Uchucay. Table 3: Frequency, Density and Importance Value Index of the woody species found in eight transects of 0.01ha in the Uchucay forest.

\begin{tabular}{|c|c|c|c|c|c|c|c|c|}
\hline Especies & Hábito & Fre. & $\begin{array}{l}\text { Fre. Rel. } \\
\quad(\%)\end{array}$ & Den. & $\begin{array}{c}\text { Den. } \\
\text { Rel. }(\%)\end{array}$ & $\begin{array}{c}\mathrm{AB} \\
\left(\mathbf{m}^{2}\right)\end{array}$ & $\begin{array}{l}\text { Dom. } \\
\text { Rel. (\%) }\end{array}$ & IVI \\
\hline Weinmannia fagaroides Kunth & Árbol & 7 & 5,147 & 78 & 14,552 & 0,848 & 13,816 & 33,515 \\
\hline Columellia oblonga Ruiz \& Pav. & Árbol & 3 & 2,206 & 8 & 1,493 & 1,502 & 24,468 & 28,167 \\
\hline Clusia flaviflora Engl. & Árbol & 6 & 4,412 & 46 & 8,582 & 0,736 & 11,993 & 24,987 \\
\hline Rudgea sp & Árbol & 7 & 5,147 & 63 & 11,754 & 0,147 & 2,395 & 19,295 \\
\hline Orepanax sp & Árbol & 1 & 0,735 & 8 & 1,493 & 0,865 & 14,082 & 16,310 \\
\hline Viburnum triphyllum Benth. & Arbusto & 6 & 4,412 & 23 & 4,291 & 0,076 & 1,238 & 9,941 \\
\hline $\begin{array}{l}\text { Myrsine coriacea (Sw.) R. Br. ex } \\
\text { Roem. \& Schult. }\end{array}$ & Árbol & 6 & 4,412 & 24 & 4,478 & 0,063 & 1,031 & 9,921 \\
\hline Myrsine andina (Mez) Pipoly & Árbol & 6 & 4,412 & 18 & 3,358 & 0,077 & 1,247 & 9,017 \\
\hline Gynoxys aff dielsiana Domke & Árbol & 4 & 2,941 & 12 & 2,239 & 0,167 & 2,726 & 7,906 \\
\hline Myrsine dependens (Ruiz \& Pav.) Spreng. & Árbol & 4 & 2,941 & 20 & 3,731 & 0,069 & 1,119 & 7,792 \\
\hline Oreocallis grandiflora (Lam.) R. Br. & Árbol & 5 & 3,676 & 13 & 2,425 & 0,094 & 1,531 & 7,633 \\
\hline Hesperomeles ferruginea (Pers.) Benth. & Árbol & 4 & 2,941 & 11 & 2,052 & 0,078 & 1,275 & 6,268 \\
\hline Clethra fimbriata Kunth & Árbol & 4 & 2,941 & 11 & 2,052 & 0,073 & 1,184 & 6,178 \\
\hline Miconia bracteolata (Bonpl.) DC. & Arbusto & 4 & 2,941 & 14 & 2,612 & 0,031 & 0,513 & 6,066 \\
\hline Gynoxys cuicochensis Cuatrec. & Arbusto & 1 & 0,735 & 8 & 1,493 & 0,223 & 3,638 & 5,865 \\
\hline Macleania hirtiflora (Benth.) A.C. Sm. & Arbusto & 4 & 2,941 & 12 & 2,239 & 0,032 & 0,517 & 5,697 \\
\hline Vallea stipularis L. f. & Árbol & 4 & 2,941 & 9 & 1,679 & 0,031 & 0,503 & 5,124 \\
\hline Ilex scopulorum Kunth & Árbol & 3 & 2,206 & 11 & 2,052 & 0,045 & 0,730 & 4,988 \\
\hline Cornus peruviana J.F. Macbr. & Árbol & 1 & 0,735 & 3 & 0,560 & 0,186 & 3,035 & 4,330 \\
\hline $\begin{array}{l}\text { Morella pubescens (Humb. \& Bonpl. ex } \\
\text { Willd.) Wilbur }\end{array}$ & Árbol & 2 & 1,471 & 3 & 0,560 & 0,140 & 2,277 & 4,307 \\
\hline Siparuna muricata (Ruiz \& Pav.) A. DC. & Árbol & 2 & 1,471 & 8 & 1,493 & 0,074 & 1,204 & 4,167 \\
\hline Verbesina latisquama S.F. Blake & Árbol & 3 & 2,206 & 7 & 1,306 & 0,028 & 0,459 & 3,971 \\
\hline Desfontainia spinosa Ruiz \& Pav. & Arbusto & 2 & 1,471 & 11 & 2,052 & 0,022 & 0,350 & 3,873 \\
\hline $\begin{array}{l}\text { Oligactis coriacea (Hieron.) } \\
\text { H. Rob. \& Brettell }\end{array}$ & Arbusto & 3 & 2,206 & 8 & 1,493 & 0,009 & 0,150 & 3,849 \\
\hline Cyathea caracasana (Klotzsch) Domin & Árbol & 2 & 1,471 & 6 & 1,119 & 0,061 & 0,996 & 3,586 \\
\hline $\begin{array}{l}\text { Tristerix longebracteatus (Desr.) } \\
\text { Barlow \& Wiens }\end{array}$ & Arbusto & 1 & 0,735 & 9 & 1,679 & 0,071 & 1,157 & 3,571 \\
\hline Ocotea infrafoveolata van der Werff & Árbol & 2 & 1,471 & 7 & 1,306 & 0,045 & 0,728 & 3,504 \\
\hline Piper andreanum C. DC. & Arbusto & 2 & 1,471 & 8 & 1,493 & 0,027 & 0,442 & 3,405 \\
\hline Gaultheria reticulata Kunth & Arbusto & 2 & 1,471 & 7 & 1,306 & 0,010 & 0,171 & 2,947 \\
\hline Axinaea macrophylla (Naudin) Triana & Árbol & 2 & 1,471 & 4 & 0,746 & 0,034 & 0,547 & 2,764 \\
\hline Miconia theaezans (Bonpl.) Cogn. & Árbol & 2 & 1,471 & 5 & 0,933 & 0,005 & 0,081 & 2,485 \\
\hline Maytenus verticillata (Ruiz \& Pav.) DC. & Árbol & 2 & 1,471 & 3 & 0,560 & 0,027 & 0,446 & 2,477 \\
\hline Lomatia hirsuta (Lam.) Diels & Arbusto & 2 & 1,471 & 4 & 0,746 & 0,008 & 0,126 & 2,343 \\
\hline $\begin{array}{l}\text { Cavendishia bracteata (Ruiz \& Pav. ex } \\
\text { J. St.-Hil.) Hoerold }\end{array}$ & Arbusto & 1 & 0,735 & 3 & 0,560 & 0,062 & 1,008 & 2,303 \\
\hline Gaultheria tomentosa Kunth & Arbusto & 2 & 1,471 & 4 & 0,746 & 0,003 & 0,055 & 2,272 \\
\hline $\begin{array}{l}\text { Oreopanax avicenniifolius (Kunth) } \\
\text { Decne. \& Planch. }\end{array}$ & Árbol & 2 & 1,471 & 3 & 0,560 & 0,003 & 0,048 & 2,078 \\
\hline
\end{tabular}




\begin{tabular}{|c|c|c|c|c|c|c|c|c|}
\hline Especies & Hábito & Fre. & $\begin{array}{l}\text { Fre. Rel. } \\
\text { (\%) }\end{array}$ & Den. & $\begin{array}{l}\text { Den. } \\
\text { Rel. }(\%)\end{array}$ & $\begin{array}{c}\mathrm{AB} \\
\left(\mathrm{m}^{2}\right)\end{array}$ & $\begin{array}{l}\text { Dom. } \\
\text { Rel. }(\%)\end{array}$ & IVI \\
\hline Styrax cordatus (Ruiz \& Pav.) A. DC. & Árbol & 2 & 1,471 & 2 & 0,373 & 0,012 & 0,192 & 2,036 \\
\hline Symplocos quitensis Brand & Árbol & 1 & 0,735 & 3 & 0,560 & 0,040 & 0,652 & 1,947 \\
\hline Podocarpus oleifolius D. Don ex Lamb. & Árbol & 1 & 0,735 & 4 & 0,746 & 0,024 & 0,391 & 1,873 \\
\hline Miconia aspergillaris (Bonpl.) Naudin & Arbusto & 1 & 0,735 & 5 & 0,933 & 0,006 & 0,094 & 1,762 \\
\hline Myrteola phylicoides (Benth.) Landrum & Arbusto & 1 & 0,735 & 5 & 0,933 & 0,003 & 0,052 & 1,721 \\
\hline Palicourea weberbaueri K. Krause & Árbol & 1 & 0,735 & 4 & 0,746 & 0,010 & 0,169 & 1,650 \\
\hline Mikania banisteriae DC. & Arbusto & 1 & 0,735 & 3 & 0,560 & 0,005 & 0,080 & 1,375 \\
\hline $\begin{array}{l}\text { Prumnopitys montana (Humb. \& Bonpl. ex } \\
\text { Willd.) de Laub. }\end{array}$ & Árbol & 1 & 0,735 & 2 & 0,373 & 0,007 & 0,114 & 1,222 \\
\hline Myrcianthes rhopaloides (Kunth) McVaugh & Árbol & 1 & 0,735 & 1 & 0,187 & 0,018 & 0,288 & 1,210 \\
\hline Duranta triacantha Juss. & Arbusto & 1 & 0,735 & 1 & 0,187 & 0,015 & 0,251 & 1,173 \\
\hline Solanum nutans Ruiz \& Pav. & Árbol & 1 & 0,735 & 2 & 0,373 & 0,004 & 0,058 & 1,166 \\
\hline Hedyosmum cumbalense H. Karst. & Árbol & 1 & 0,735 & 2 & 0,373 & 0,002 & 0,032 & 1,140 \\
\hline Stylogyne sp & Árbol & 1 & 0,735 & 2 & 0,373 & 0,001 & 0,017 & 1,125 \\
\hline Bejaria resinosa Mutis ex L. f. & Arbusto & 1 & 0,735 & 1 & 0,187 & 0,005 & 0,082 & 1,004 \\
\hline Myrcianthes sp & Árbol & 1 & 0,735 & 1 & 0,187 & 0,005 & 0,082 & 1,004 \\
\hline Persea brevipes Meisn. & Árbol & 1 & 0,735 & 1 & 0,187 & 0,004 & 0,063 & 0,985 \\
\hline Prunus huantensis Pilg. & Árbol & 1 & 0,735 & 1 & 0,187 & 0,003 & 0,046 & 0,968 \\
\hline Disterigma $\mathrm{sp}$ & Arbusto & 1 & 0,735 & 1 & 0,187 & 0,001 & 0,020 & 0,942 \\
\hline Geissanthus sp & Árbol & 1 & 0,735 & 1 & 0,187 & 0,001 & 0,012 & 0,933 \\
\hline Miconia hexamera Wurdack & Árbol & 1 & 0,735 & 1 & 0,187 & 0,001 & 0,012 & 0,933 \\
\hline Weinmannia stenocarpa Killip \& A.C. Sm. & Árbol & 1 & 0,735 & 1 & 0,187 & 0,000 & 0,008 & 0,930 \\
\hline TOTAL & & 136 & 100 & 536 & 100 & 6,141 & 100,000 & 300,000 \\
\hline
\end{tabular}

como: Prumnopitys montana; Podocarpus oleifolius, y Styrax cordatus, que seguramente en el pasado fueron importantes pero debido a la deforestación sus poblaciones están en decremento y a pesar de tener una amplia distribución son consideradas como especies amenazadas.

\subsection{Percepción local biocultural}

Las respuestas de las entrevistas reflejan un conocimiento general sobre las especies del bosque andino en función mayoritariamente del uso maderable o de alimentación que se puede obtener de dichas especies. Todos los entrevistados $(n=30)$ confirman que el paisaje del BTMN ha cambiado radicalmente por la continua tala de árboles grandes y de fuste rectilíneo para usar en construcción o como postes de linderos de propiedades, así como también por la implementación de prácticas agrícolas (siembras de altura) o ganaderas (pastoreo de ganado vacuno y lanar) de pequeña escala, asumiendo modelos de subsistencia y sin la utilización de agroquímicos. La edad media en Uchucay es de 54 años. Todos los entrevistados fueron campesinos de la tercera edad quienes indicaron que los jóvenes ya no están haciendo las labores del cam- po, sino que prefieren ir a la ciudad cercana de Gualaceo o a la capital de la provincia, Cuenca, para estudios o trabajos en las zonas urbanas. Este flujo migratorio campociudad se ha mantenido durante las últimas décadas y se ha acentuado en algunas zonas de las provincias del austro ecuatoriano (Donoso-Correa \& Sarmiento, 2019).

El $70 \%$ de los entrevistados recuerdan la abundante fauna que caracterizaba al sector y que con la desaparición de la masa forestal ya no se los encuentra con tanta frecuencia. En particular hacen referencia al oso de anteojos (Tremartus ornatus), el 'león' americano (Puma concolor), la danta de altura (Tapirus pinchaque), el lobo de páramo (Ducysion culpaeus), el venado grande (Odocoileus virginianus spp ustus), el venado chico (Mazama americana) y el venado cabrío o soche (Mazama rufina). Incluso los conejos (Sylvilagus brassiliensis spp andinus) y los topitos de campo (Microtis sp) se citan como escasos. También notan la reducción en avistamiento de pájaros otrora comunes, como el tucán andino (Andigena hypoglauca, Ornithorynchus erytropygius y Ramphastinus andinus) y aves de rapiña como guarros (Geranoaetus melanoleucus), quriquingues (Phalcoboenus carunculatus) y gavilanes (Buteo polyosoma). Un grupo definitivamente no visto recientemente es el de las ranas de monta- 
ña (Atelopus cf. nanay). Es incierto si su ausencia se debe a la falta de aves, a la transformación del paisaje rural o a la intensa deforestación soportada por los BTMN de esta región. Tan sólo tres entrevistados refieren a la presencia incrementada de animales domésticos que merodean la zona de relicto, incluyendo gatos y perros cimarrones. En dos de las visitas al área de estudio se constató evidencia directa de pastoreo con ganado vacuno dentro de las zonas boscosas.

Sin embargo, el $82 \%$ de las respuestas sobre la ocupación del lugar y las actividades parecen ser influenciadas por el miedo a identificar al encuestador con censores estatales que podrían afectar su declaración de impuestos. Por lo tanto, la información obtenida sobre el número de animales o sobre qué tipo de producción ejercen se ha mantenido sin analizar para evitar prejuicios y falsos estadísticos. Todos los encuestados afirmaron que la desaparición de especies se debe al abuso prolongado de muchas generaciones, pero no existió consenso en que si es ancestral o no. Curiosamente, los residentes en el sitio no son indígenas Kañary ni Shwar, quienes habitan las provincias aledañas hacia la serranía y hacia la amazonía respectivamente. Los habitantes de las localidades vecinas a Uchucay en la mancomunidad de El Collay no se identifican como indígenas, ni exhiben indigenismo en sus prácticas de uso de la tierra mencionadas por los encuestados. Estas prácticas del campesinado son típicas del paisaje rural híbrido con prácticas mediterráneas y americanas, tanto en la chakra como en el pajonal. La descripción de las preguntas de la encuesta se incluye en el Anexo 2.

\section{Discusión y conclusión}

La diversidad registrada en este remanente se considera relevante, al compararlo con otros sitios con similar vegetación, por ejemplo, en un bosque montano de Perú localizado entre 2200 y 2900 m.s.n.m. en una superficie de 0,25 ha, registraron 41 especies, 33 géneros y 25 familias (Rasal-Sánchez et al., 2011), frente a 57 especies pertenecientes a 47 géneros y 32 familias registradas en 0,08 ha de este estudio. Por otra parte, en bosques montanos localizados en la provincia de Loja al sur del Ecuador, en 132 parcelas de 0,04 ha, Cabrera et al. (2019) registraron 164 especies pertenecientes a 52 familias botánicas.

Es conocido que los BTMN representan uno de los ecosistemas con mayor diversidad (Myers et al., 2000), por lo cual los patrones de diversidad y composición florística son muy variables y dependen de muchos factores, entre los cuales la ubicación geoespacial juega un papel preponderante. El área de estudio de Uchucay se encuentra espacializada en la zona de confluencia de dos formaciones geológicas: la formación de los Andes del norte, en donde existe la dominancia de volcanes activos de gran altitud y la formación de los Andes del sur, sin la presencia de volcanes y en donde sus montañas pocas veces superan los $4000 \mathrm{~m}$ de altitud (Hall, 1977). Como resultado, los BTMN y su vegetación nativa guardan elementos florísticos provenientes tanto del norte (e.g.,
Symplocos y Hedyosmum) cuanto de elementos florísticos provenientes del sur (e.g., Podocarpus y Oreocallis) (Jorgensen et al., 1995); esto determina que la composición florística cambie drásticamente de un sitio a otro, aunque las diferencias fisonómicas y estructurales no sean significativas.

En este estudio si analizamos la diversidad y composición de cada uno de los transectos notamos que estos varían en relación con factores topográficos locales; de esta manera vemos que los sitios con mayor pendiente muestran mayor riqueza de especies y una alta concentración de individuos, normalmente estos sitios corresponden a ladera y crestas (Tabla 2). Por lo tanto, la heterogénea distribución de los factores climáticos a microescala facilita la diversificación de las especies (Robertson et al., 2010). Contrariamente en los sitios planos correspondientes a valles se concentran pocas especies con individuos de gran tamaño que tienen mayor área basal tal como se observa en los transectos 2 y 3 (Tabla 2).

En Uchucay, las especies dominantes son: Weinmannia fagaroides, Columellia oblonga y Clusia flaviflora, mientras que en Loja, al sur del Ecuador, en BTMN de similares características las especies dominantes son: Schefflera ferruginea, Prumnopitys montana, Podocarpus oleifolius y Guarea kunthiana (Cabrera et al., 2019); $\mathrm{y}$, por otro lado, un estudio del Perú indica que las especies dominantes son Nectandra sp, Myrsine latifolia y Chrysophyllum sp (Rasal-Sánchez et al., 2011), lo cual confirma la gran heterogeneidad florística que presentan los BTMN. Entre las especies representativas de los BTMN de la región sur del Ecuador se encuentra Podocarpus oleifolius que, pese a que presenta una amplia distribución en los países andinos (Vicuña-Miñano, 2005; Arroyo et al., 2008) se encuentra amenazada; por tanto, encontrar relictos de esta especie cada vez se torna más difícil (Figuras 6a y 6b) e incluso en áreas protegidas, como el Parque Nacional Podocarpus, quedan pocos individuos maduros de crecimiento viejo (Ambulay, 2008).

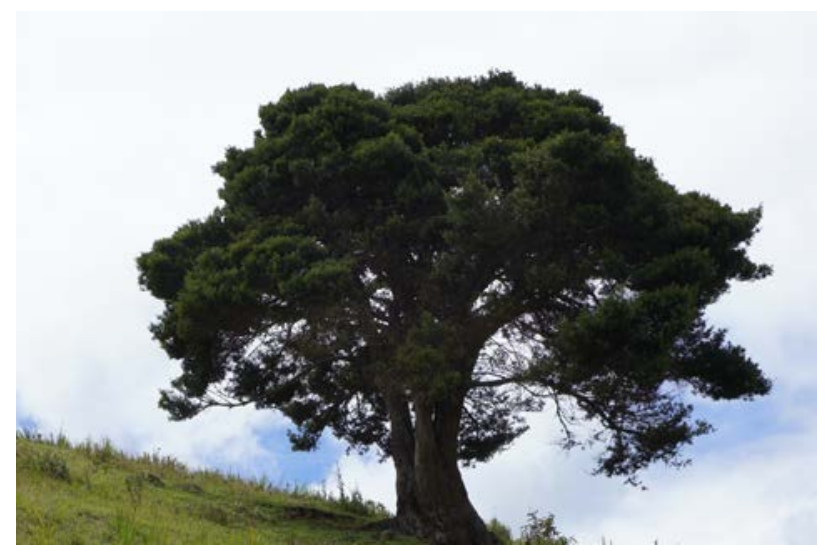

Figura 6a: Ejemplo de microrefugio biocultural formado por el solitario árbol de Romerillo (Podocarpus oleifolius) en las immediaciones de la reserva comunitaria de Uchucay.

Figure 6a: Example of the biocultural microrefugium formed by the lonely tree of 'Romerillo'(Podocarpus oleifolius) in the vicinity of the Uchucay Community Reserve. 


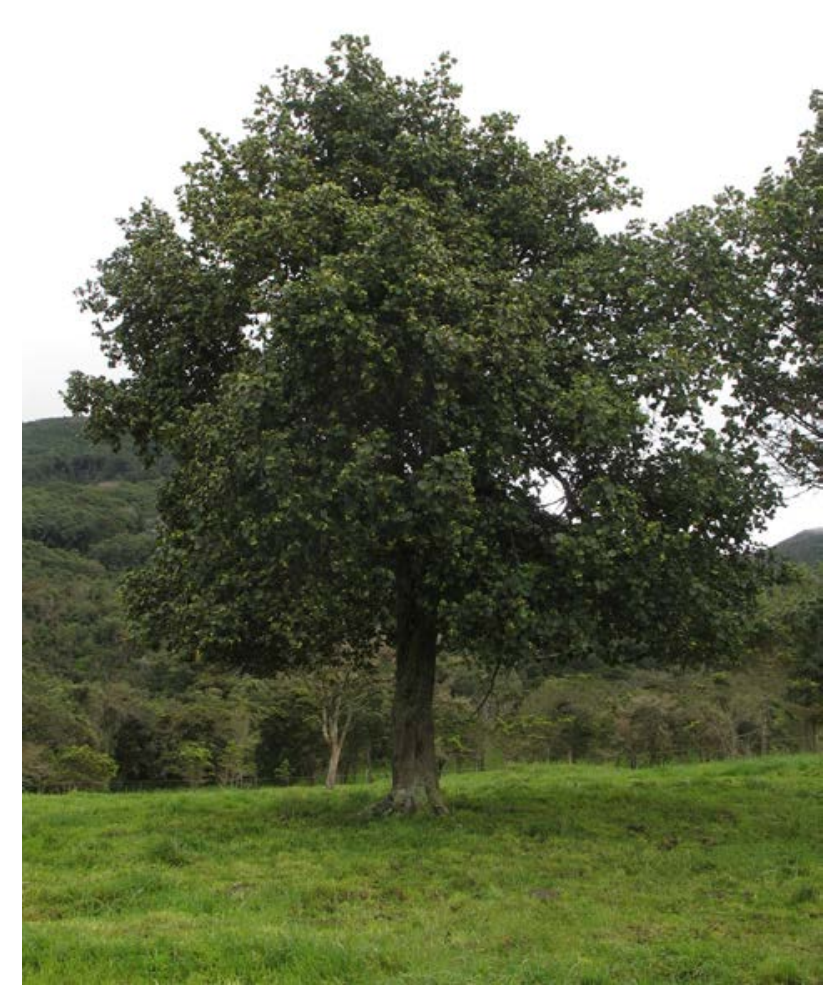

Figura 6b: Otro solitario árbol de Guandera (Clusia flaviflora) como testamento de la potencialidad que existe al considerarlos árboles nodriza para la regeneración del bosque andino en microrefugios bioculturales patrimoniales.

Figure 6b: Another lonely tree of 'Guandera'(Clusia faviflora) as testament of the potential for regeneration that exists when

considered as nursery tree for Andean forest restoration in biocultural heritage microrefugia.

Estos resultados nos llevan a concluir que aún existen vacíos de información sobre estos ecosistemas, lo que, de acuerdo a Bruijnzeel et al., (2010) se debe a tres factores: 1. información inadecuada sobre la distribución espacial, la riqueza biológica y la variación ecológica de los BTMN a escala continental, regional, de paisaje y local; 2. información limitada sobre la dinámica climática, de nutrientes y del carbono, y su relación con la productividad, la resiliencia, el potencial de restauración y el estado de conservación de los BTMN; y 3. información incompleta sobre los efectos del cambio en el uso de la tierra y del clima en la biodiversidad y las dinámicas ecosistémicas de los bosques montanos. Uno de nosotros (Sarmiento, 2012) enfatiza que un cuarto factor importante es la hibridación de las prácticas de manejo territoriales al deforestar los BTMN y favorecer la extensión del páramo pajonal.

Una estrategia para llenar estos vacíos de conocimiento es establecer Sitios de Investigaciones Ecológicas de Largo Plazo (SIELP), con sistemas de parcelas permanentes, centradas en el estudio de la ecología y el funcionamiento de los BTMN en gradientes ambientales, particularmente los altitudinales (Pinto et al., 2008), además de investigar el valor cultural y los beneficios que las comu- nidades otorgan a estos paisajes. Estos parámetros sin duda servirán para elaborar políticas de manejo enfocadas a la conservación de los BTMN como microrefugios bioculturales patrimoniales y los beneficios que las comunidades puedan obtener en el sitio al mantenerlo con la cobertura vegetal arbórea. Aspiramos a que con la herborización de las flórulas de orquídeas (Ecuagénera, 2017) y el levantamiento de árboles de la reserva comunitaria de Uchucay (este trabajo), se logre incrementar el nivel de conciencia ambiental de las personas que viven en el área o que dependen directamente de ella, y que se favorezca la gobernanza de conservación de la mancomunidad El Collay y la reserva comunitaria de Uchucay como microrefugios de diversidad biocultural en los Andes.

\section{Agradecimientos}

El trabajo se presentó como un afiche en la Conferencia Internacional sobre Vegetación Pasada, Cambio Climático y Conservación de Montañas, organizada por el proyecto VULPES en Cuenca, en Marzo 2019. Agradecemos al proyecto VULPES (Project ID: ANR-15- MASC0003) con el auspicio del Belmont Forum, que facilitó la colaboración inter-universitaria azuaya y el Colaboratorio de Montología Neotropical. Gracias al municpio de Guadalfeo y a los comuneros de Uchucay. Gracias también a tres revisores anónimos que perfeccionaron el estilo y mejoraron la presentación de los datos y especialmente a Teodoro Lasanta Martínez que llevó la tarea editorial de forma ejemplar.

\section{Referencias}

APG IV., 2016. An update of the Angiosperm Phylogeny Group classification for the orders and families of flowering plants: APG IV. Botanical Journal of the Linnean Society, 181: 1-20. https://doi.org/10.1111/boj.12385

Ambulay, I.M., 2008. Etnobotánica en las comunidades campesinas Cuyas - Cuchayo, Joras y Suyupampa del bosque de Cuyas, Ayabaca - Piura, 2006. Tesis Lic. Biología. Universidad Nacional de Piura, Perú.

Arroyo, S.J., Rodríguez, E.F., Leiva, S., Zapata, M. \& Mora L., 2008. El bosque relicto de Cachil (Provincia Gran Chimú, Departamento La Libertad, Perú), un ecosistema que necesita planes de conservación urgente. Arnaldoa, 15: 289-296.

Bonifaz, C. \& Cornejo, X., 2002. Proteaceae. In: G. Harling \& L. Andersson (eds), Flora of Ecuador 69: 3-48 University of Göteborg; Riksmuseum, Göteborg, Stockholm.

Bruijnzeel, L.A., Kapelle, M., Mulligan, M. \& Scatena, F.N., 2010 Tropical montane cloud forests: state of knowledge and sustainability perspectives in a changing world. Pp. 691-740. En: L.A. Bruijnzeel, F.N. Scatema \& L.S. Hamilton (eds.), Tropical montane cloud forests: state of knowledge and sustainability perspectives in a changing world. Cambridge University Press. https://doi.org/10.1017/ CBO9780511778384.074

Bruijnzeel, L.A., Mulligan, M. \& Scatena, F., 2011. Hydrometeorology of tropical montane cloud forests: emerging patterns. Hydrological Processes, 25: 465-498. https://doi. org/10.1002/hyp.7974 
Cabrera, O., Benítez, A., Cumbicus, N., Naranjo, C., Ramón, P., Tinitana, F. \& Escudero, A., 2019. Geomorphology and Altitude Effects on the Diversity and Structure of the Vanishing Montane Forest of Southern Ecuador. Diversity, 11, 32; https://doi.org/10.3390/d11030032

Carmona-Galindo, V. \& Carmona, T., 2013. La diversidad de los análisis de diversidad. Bioma, 14: 20-28

Condit, R., Ashton, P.S., Baker, P., Bunyavejvhewin, S., Gunatilleke, S., Gunatilleke, N., Hubbell, S.P., Foster, R.B., Itoh, A., la Frankie, J.Y., Lee, H.S., Losos, E., Manokaran, N., Sukumar, R., \& Yamakura, T., 2000. Spatial patterns in the distribution of tropical tree species. Science, 288. https:// doi.org/10.1126/science.288.5470.1414

Cuesta, F., Peralvo, M. \& Valarezo, N., 2009. Los bosques montanos de los Andes Tropicales. Quito, Lima, La Paz: Programa Regional Ecobona-In

Donoso-Correa, M.E \& Sarmiento, F.O., 2019. Geospatial Memory and Joblesness interpolated: International migration oxymora in the city of Biblián, Southern Ecuador. American Journal of Geographic Information System, 8(2): 60-88.

Ecuagénera, 2017. Uchucay: Reserva Comunitaria. Evelyn Huck, Omar Caizaluisa y Gloria Aguilar (Editores). GAD municipal del Cantón Gualaceo/Géneros Ecuatorianos Ecuagénera Orquídeas del Ecuador. Gualaceo: Imprenta Alcaldía.

Fagerström, K., 1975. Columelliaceae. In: G. Harling \& B. Sparre (eds), Flora of Ecuador 4: 1-5. University of Göteborg; Riksmuseum, Göteborg, Stockholm.

Field Museum Herbarium, 2019. Visitado por última vez en Feb., 02, 2019. https://plantidtools.fieldmuseum.org/es/ $\mathrm{rrc} / 5581$

Gentry, A.H., 1982. Patterns of Neotropical Plant Species Diversity. Evolutionary Biology, 15: 1-84. https://doi. org/10.1007/978-1-4615-6968-8 1

Gustafsson, C., 1992. Clethraceae. In: G. Harling \& L. Andersson (eds), Flora of Ecuador, 45: 1-26 University of Göteborg; Riksmuseum, Göteborg, Stockholm.

Hall, P.M., 1977. El volcanismo en el Ecuador. Quito, Ecuador.

Harling, G., 1999. Cunoniaceae. In: G. Harling \& L. Andersson (eds), Flora of Ecuador, 61: 1-74 University of Göteborg; Riksmuseum, Göteborg, Stockholm.

Herbario Azuay., 2019. Visitado por última vez en Feb., 14, 2019. http://www.uazuay.edu.ec/HerbarioAzuay/.

Hill, M.O., 1973 Diversity and Evenness: A Unifying Notation and Its Consequences, Ecology, 54 (2): 427-432. https://doi. org/10.2307/1934352

Hofstede, R., Lips, J., Jongsma, W. \& Servir, Y., 1998. Geografía, Ecología y Forestación de la Sierra Alta del Ecuador. Ediciones Abya-Yala. Páginas 52-69. Quito, Ecuador.

Jarvis, A. \& Mulligan, M., 2011. The climate of cloud forests. Hydrological Processes, 25: 327-343. https://doi. org/10.1002/hyp.7847

Jørgensen, P.M., Ulloa-Ulloa, C., Madsen, J.E., \& Valencia, R., 1995. A floristic analysis of the high Andes of Ecuador. In: Biodiversity and Conservation of Neotropical Montane Forests; Churchill, S.P., Balslev, H., Forero, E., \& Luteyn, J.L., Eds.; New York Bot. Gard: New York, NY, USA, pp. 221-238.

Jorgensen, P.M., \& León-Yánez, S., 1999. Catalogue of the vascular plants of Ecuador. Missouri Botanical Garden, 1181 pp., St. Louis, Missouri.

JSTOR ORG. Global Plants. 2019. Revisado por última vez en Feb., 16, 2019 https://www.jstor.org/

Kuijt, J., 1986. Loranthaceae. In: G. Harling \& B. Sparre (eds), Flora of Ecuador, 24: 113-194 University of Göteborg; Riksmuseum, Göteborg, Stockholm.

Ledo, A., Montes, F. \& Condes, S., 2009. Species dynamics in a montane cloud forest: Identifying factors involved in changes tree diversity and functional characteristics. Forest Ecology and Management, 258: S75-S84.

León-Yánez, S., Valencia, R., Pitman, N., Endara, L., Ulloa Ulloa, C. \& Navarrete, H., 2011. Libro rojo de las plantas endémicas del Ecuador, segunda edición. Publicaciones del Herbario QCA, Pontificia Universidad Católica del Ecuador, Quito.

Luteyn, J., 1996. Ericaceae. In: G. Harling \& L. Andersson (eds), Flora of Ecuador, 54: 253-285 University of Göteborg; Riksmuseum, Göteborg, Stockholm.

Mathez-Stiefel, S.L., Peralvo, M., Báez, S., Rist, S., Buytaert, W., Cuesta, F., Baez, S. ... \& Llambí, L.D., 2017. Research priorities for the conservation and sustainable governance of Andean forest landscapes. Mountain Research and Development, 37(3): 323-340. https://doi.org/10.1659/ MRD-JOURNAL-D-16-00093.1

McGarical, K. \&. Cushman, A., 2002. The Gradient Concept of Landscape Structure: Or, Why are there so Many Patches, Department of Natural Resources Conservation, University of Massachusetts, Amherst, MA, USA.

Mostacedo, B. \& Fredericksen, T., 2000. Manual de métodos básicos de muestreo y análisis en Ecología vegetal. BOLFOR. 84 pp. Santa Cruz, Bolivia.

Mulligan M., 2011. Modeling the tropics-wide extent and distribution of cloud forest and cloud forest loss, with implications for conservation priority. Pg. 14-38 en L. A. Bruijnzeel, F. N. Scatena \& L. S. Hamilton (eds.), Tropical montane cloud forests: Science for conservation and management, Cambridge y New York: Cambridge University Press. https://doi.org/10.1017/CBO9780511778384.004

Myers, N., Mittermeier, R.A., Mittermeier, C.G., da Fonseca, G.A.B., \& Kent, J., 2000. Biodiversity hotspots for conservation priorities. Nature, 403: 853-858. https://doi. org/10.1038/35002501

New York Botanical Garden. 2019. Revisado por última vez en Feb., 16, 2019 http://sciweb.nybg.org/science2/VirtualHerbarium.asp.

Pennington, D.W., Potting, J., Finnveden, G., Lindeijer, E., Jolliet, O., Rydberg, T. \& Rebitzer, G., 2004. Life cycle assessment Part 2: Current impact assessment practice. Environment International, 30(5): 721-739. https://doi. org/10.1016/j.envint.2003.12.009

Pinto, E., Pérez, A.J., Ulloa Ulloa, C., \& Cuesta, F., 2018. Árboles representativos de los bosques montanos del noroccidente de Pichincha, Ecuador. CONDESAN, Quito, Ecuador.

Rasal-Sánchez, M., Troncos Castro, J., Lizano Durán, C., Parihuamán Granda, O., Quevedo Calle, D., Rojas Idrogo, C. \& Delgado Paredes, G., 2011. Características edáficas y composición florística del bosque estacionalmente seco La Menta y Timbes, region Piura, Perú. Ecología Aplicada, 10(2). http://dx.doi.org/10.21704/rea.v10i1-2.414

Renner, S. \& Hausner, G., 1996. Siparunaceae. In: G. Harling \& L. Andersson (eds), Flora of Ecuador, 59: 3-98 University of Göteborg; Riksmuseum, Göteborg, Stockholm.

Robertson, A., Malhi, Y., Farfan-Amezquita, F., Araga, L.E., Silva Espejo, J.E. \& Robertson, M., 2010. Stem respiration in tropical forests along an elevation gradient in the Amazon and Andes. Global Change Biology, 16: 3193-3204. https:// doi.org/10.1111/j.1365-2486.2010.02314.x

Robinson, H., 2007a. Compositae-Heliantheae. In: G. Harling \& L. Andersson (eds), Flora of Ecuador, 77 (2): 170-195. University of Göteborg; Riksmuseum, Göteborg, Stockholm.

Robinson, H., 2007b. Compositae-Eupatorieae. In: G. Harling \& C. Persson (eds), Flora of Ecuador, 83: 206-294. University of Göteborg; Riksmuseum, Göteborg, Stockholm. 
Robinson, H., 1978. Compositae-Liabeae. In: G. Harling \& B. Sparre (eds), Flora of Ecuador, 8: 51-56. University of Göteborg; Riksmuseum, Göteborg, Stockholm.

Romoleroux, K., 1996. Rosaceae. In: G. Harling \& L. Andersson (eds), Flora or Ecuador, 56: 3-156 University of Göteborg; Riksmuseum, Göteborg, Stockholm.

Sarmiento, F.O., 2016. Identity, imaginaries and ideality: understanding the biocultural landscape of the Andes through the iconic Andean lapwing (Vanellus resplendens). Revista Chilena de Ornitología, 22(1): 38-50

Sarmiento, F.O., 2012. Contesting Páramo: Critical Biogeography of the Northern Andean Highlands. Charlotte: Kona Publishing.

Sarmiento, F.O., 2001. Ecuador. Pp 497-548. In: Kapelle, M. and A. Brown. Bosques Nublados del Neotrópico. National Institute of Biodiversity (InBIO): San José, Costa Rica. 698pp.

Sarmiento, F.O., 1988. Antología Ecológica del Ecuador: Desde la Selva hasta el Mar. Publicaciones del Museo Ecuatoriano de Ciencias Naturales. Quito: Casa de la Cultura Ecuatoriana.

Sarmiento, F.O., Vázques, A., Aguilar, G., Cheddadi, R., Bush, M., Donoso, M., Palacios, E. \& Kong, I., 2018. Trees Microrefugia and Community-based Conservation in Tropandean Mountainscapes: A Bio-Cultural Approach for Heritage Management of "El Collay" Protected Forest in Southeastern Ecuador. Satoyama Review, 4(1): 95-109.

Sarmiento, F.O. \& Viteri X., 2015. Discursive Heritage: Sustaining Andean Cultural Landscapes Amidst Environmental Change. Taylor, K., A. St Clair \& N.J. Mitchell (Eds). Conserving Cultural Landscapes: Challenges and New Directions. Routledge, New York.
Sierra, R., Cerón, C.E., Palacios, W. \& Valencia, R., 1999. Propuesta preliminar de un sistema de clasificación de vegetación para el Ecuador continental. Páginas 29-54. Proyecto INEFAN/GEF-BIRF y EcoCiencia, Quito.

Ståhl, B., 1991. Symplocaceae. In: G. Harling \& L. Andersson (eds), Flora of Ecuador, 43: 3-44 University of Göteborg; Riksmuseum, Göteborg, Stockholm.

Taylor, Ch., 1999. Rubiaceae (Tribe Coussareeae). In: G. Harling \& B. Sparre (eds), Flora of Ecuador 62: 245-314. University of Göteborg; Riksmuseum, Göteborg, Stockholm.

Todzia, C., 1990. Chloranthaceae. In: G. Harling \& B. Sparre (eds), Flora of Ecuador, 40: 1-32. University of Göteborg; Riksmuseum, Göteborg, Stockholm.

TROPICOS, 2019. Missouri Botanical Garden. Visitado por última vez en Feb., 12, 2019. http://tropicos.org/

Tryon, R., 1986. Cyatheaceae. In: G. Harling \& L. Andersson (eds), Flora of Ecuador, 27: 19-56 University of Göteborg; Riksmuseum, Göteborg, Stockholm.

Valencia, R.N., Pitman, S., León-Yánez \& Jorgensen, P.M., 2000. Libro rojo de las plantas endémicas del Ecuador 2000. Herbario QCA, Pontificia Universidad Católica del Ecuador, Quito. 489 pp.

Velasco-Linares, P. \& Vargas, O., 2007. Problemática de los Bosques Altoandinos. En: Vargas O. (ed.). Estrategias para la Restauración Ecológica del Bosque Altoandino: El Caso de la Reserva Forestal Municipal de Cogua, Cundinamarca. Pág. 33 a 45. Grupo de Restauración Ecológica. Departamento de Biología. Universidad Nacional de Colombia. Bogotá, Colombia.

Vicuña-Miñano, E.E., 2005. Las Podocarpáceas de los bosques montanos del noroccidente. Revista Peruana de Biología, 12: 283-288. https://doi.org/10.15381/rpb.v12i2.2400

Anexo 1: Inventario General de especies.

Appendix 1: General plant species inventory

\begin{tabular}{|c|c|c|c|}
\hline Familia & Especie & Origen & $\begin{array}{l}\text { Categoría } \\
\text { de amenaza }\end{array}$ \\
\hline AQUIFOLIACEAE & Ilex scopulorum Kunth & $\mathrm{N}$ & \\
\hline ARALIACEAE & Oreopanax avicenniifolius (Kunth) Decne. \& Planch. & $\mathrm{E}$ & NT \\
\hline ARALIACEAE & Orepanax sp & $\mathrm{N}$ & \\
\hline ASTERACEAE & Gynoxys cuicochensis Cuatrec. & $\mathrm{N}$ & \\
\hline ASTERACEAE & Gynoxys aff dielsiana Domke & $\mathrm{N}$ & VU \\
\hline ASTERACEAE & Mikania banisteriae DC. & $\mathrm{N}$ & \\
\hline ASTERACEAE & Oligactis coriacea (Hieron.) H. Rob. \& Brettell & $\mathrm{N}$ & \\
\hline ASTERACEAE & $\begin{array}{l}\text { Verbesina } \\
\text { latisquama S.F. Blake }\end{array}$ & $\mathrm{N}$ & \\
\hline CAPRIFOLIACEAE & Viburnum triphyllum Benth. & $\mathrm{N}$ & \\
\hline CELASTRACEAE & Maytenus verticillata (Ruiz \& Pav.) DC. & $\mathrm{N}$ & \\
\hline CHLORANTHACEAE & Hedyosmum cumbalense H. Karst. & $\mathrm{N}$ & \\
\hline CLETHRACEAE & Clethra fimbriata Kunth & $\mathrm{N}$ & \\
\hline CLUSIACEAE & Clusia flaviflora Engl. & $\mathrm{N}$ & \\
\hline COLUMELLIACEAE & Columellia oblonga Ruiz \& Pav. & $\mathrm{N}$ & \\
\hline CORNACEAE & Cornus peruviana J.F. Macbr. & $\mathrm{N}$ & \\
\hline CUNONIACEAE & Weinmannia fagaroides Kunth & $\mathrm{N}$ & \\
\hline CUNONIACEAE & Weinmannia stenocarpa Killip \& A.C. Sm. & $\mathrm{N}$ & \\
\hline
\end{tabular}


EL MICROREFUGIO DE UCHUCAY: UN RELICTO DE BOSQUE INTERANDINO CON UNA IMPORTANTE RIQUEZA...・15

\begin{tabular}{|c|c|c|c|}
\hline Familia & Especie & Origen & $\begin{array}{l}\text { Categoría } \\
\text { de amenaza }\end{array}$ \\
\hline CYATHEACEAE & Cyathea caracasana (Klotzsch) Domin & $\mathrm{N}$ & \\
\hline ELAEOCARPACEAE & Vallea stipularis L. f. & $\mathrm{N}$ & \\
\hline ERICACEAE & Bejaria resinosa Mutis ex L. f. & $\mathrm{N}$ & \\
\hline ERICACEAE & Cavendishia bracteata (Ruiz \& Pav. ex J. St.-Hil.) Hoerold & $\mathrm{N}$ & \\
\hline ERICACEAE & Disterigma sp & $\mathrm{N}$ & \\
\hline ERICACEAE & Gaultheria reticulata Kunth & $\mathrm{N}$ & \\
\hline ERICACEAE & Gaultheria tomentosa Kunth & $\mathrm{N}$ & \\
\hline ERICACEAE & Macleania hirtiflora (Benth.) A.C. Sm. & $\mathrm{N}$ & \\
\hline LAURACEAE & Ocotea infrafoveolata van der Werff & $\mathrm{N}$ & \\
\hline LAURACEAE & Persea brevipes Meisn. & $\mathrm{N}$ & \\
\hline LOGANIACEAE & Desfontainia spinosa Ruiz \& Pav. & $\mathrm{N}$ & \\
\hline LORANTHACEAE & Tristerix longebracteatus (Desr.) Barlow \& Wiens & $\mathrm{N}$ & \\
\hline MELASTOMATACEAE & Axinaea macrophylla (Naudin) Triana & $\mathrm{N}$ & \\
\hline MELASTOMATACEAE & Miconia aspergillaris (Bonpl.) Naudin & $\mathrm{N}$ & \\
\hline MELASTOMATACEAE & Miconia bracteolata (Bonpl.) DC. & $\mathrm{N}$ & \\
\hline MELASTOMATACEAE & Miconia hexamera Wurdack & E & VU \\
\hline MELASTOMATACEAE & Miconia theaezans (Bonpl.) Cogn. & $\mathrm{N}$ & \\
\hline MYRICACEAE & Morella pubescens (Humb. \& Bonpl. ex Willd.) Wilbur & $\mathrm{N}$ & \\
\hline MYRISTICACEAE & Stylogyne sp & $\mathrm{N}$ & \\
\hline MYRTACEAE & Myrcianthes rhopaloides (Kunth) McVaugh & $\mathrm{N}$ & \\
\hline MYRTACEAE & Myrcianthes sp & $\mathrm{N}$ & \\
\hline MYRTACEAE & Myrteola phylicoides (Benth.) Landrum & $\mathrm{N}$ & \\
\hline PIPERACEAE & Piper andreanum C. DC. & $\mathrm{N}$ & \\
\hline PODOCARPACEAE & Podocarpus oleifolius D. Don ex Lamb. & $\mathrm{N}$ & NT \\
\hline PODOCARPACEAE & Prumnopitys montana (Humb. \& Bonpl. ex Willd.) de Laub. & $\mathrm{N}$ & VU \\
\hline PRIMULACEAE & Geissanthus sp & $\mathrm{N}$ & \\
\hline PRIMULACEAE & Myrsine andina (Mez) Pipoly & $\mathrm{N}$ & \\
\hline PRIMULACEAE & Myrsine coriacea (Sw.) R. Br. ex Roem. \& Schult. & $\mathrm{N}$ & \\
\hline PRIMULACEAE & Myrsine dependens (Ruiz \& Pav.) Spreng. & $\mathrm{N}$ & \\
\hline PROTEACEAE & Lomatia hirsuta (Lam.) Diels & $\mathrm{N}$ & \\
\hline PROTEACEAE & Oreocallis grandiflora (Lam.) R. Br. & $\mathrm{N}$ & \\
\hline ROSACEAE & Hesperomeles ferruginea (Pers.) Benth. & $\mathrm{N}$ & \\
\hline ROSACEAE & Prunus huantensis Pilg. & $\mathrm{N}$ & \\
\hline RUBIACEAE & Palicourea weberbaueri K. Krause & $\mathrm{N}$ & \\
\hline RUBIACEAE & Rudgea sp & $\mathrm{N}$ & \\
\hline SIPARUNACEAE & Siparuna muricata (Ruiz \& Pav.) A. DC. & $\mathrm{N}$ & \\
\hline SOLANACEAE & Solanum nutans Ruiz \& Pav. & $\mathrm{N}$ & \\
\hline STYRACACEAE & Styrax cordatus (Ruiz \& Pav.) A. DC. & $\mathrm{N}$ & \\
\hline SYMPLOCACEAE & Symplocos quitensis Brand & $\mathrm{N}$ & \\
\hline VERBENACEAE & Duranta triacantha Juss. & $\mathrm{N}$ & \\
\hline
\end{tabular}


Anexo 2: Cuestionario guía para entrevistas de campo en la reserva comunitaria de Uchucay. Appendix 2: Survey questionnaire for field interviews in the Uchucay Community Reserve.

\section{COLABORATORIO DE MONTOLOGÍA NEOTROPICAL}

VULPES: VULnerabilidad de las Poblaciones bajo Escenarios extremoS Cuestionario para los residentes locales y los administradores

\section{Preguntas introductorias}

- 1. ¿Actualmente o en el pasado ha vivido en la zona?

- 2. ¿Cuánto tiempo usted ha vivido como un campesino?

- 3. ¿Usted ha cultivado siempre el sitio en la montaña? Si no, ¿dónde?

\section{Los medios de subsistencia de montaña}

- 4. ¿Con qué frecuencia va al bosque? ¿Qué duración tiene su visita allí?

- 5. ¿Qué condiciones determinan cuándo usted elige visitar?

- 6. ¿Siempre cultiva durante estas condiciones favorables?

- 7. ¿Qué papel juega la especie endémica (Pino andino) en su comunidad?

- 8. ¿Cómo percibe el estado de biodiversidad y agricultura sostenible en el área?

- 9. Si se siente cómodo compartiendo, es cultivar su ocupación de tiempo completo, o ¿tiene otros trabajos así?

- 10. ¿Qué retos y amenazas percibe que afectan a su forma de vida en la montaña?

\section{Mentalidad ambiental}

- 11. ¿Cómo percibe el entorno natural el estado de su país?

- 12. ¿Cómo percibe usted la relación de la naturaleza y las áreas protegidas?

- 13. ¿Sabe en dónde están las áreas protegidas? ¿Dónde están las áreas que identifican como medio importante para la agricultura? ¿Dónde poder abrir su parcela para cultivar?

- 14. ¿En estas fotografías, se puede identificar las especies comunes que se utilizan a menudo para las cercas o para carbón de leña? ¿Cuáles han sido sus experiencias con la conservación de la biodiversidad en estos lugares?

\section{Microrefugios y gestión ambiental}

- 15. ¿Está familiarizado con las especies introducidas?

- 16. ¿Utiliza recursos de las especies introducidas? ¿Si es así, explicar qué tipo de usos fueron?

- 17. ¿Ha sido capaz de interactuar con el personal del área protegida? Si es así, ¿estás dispuesto a describir esta interacción?

- 18. ¿Cuáles son sus pensamientos iniciales sobre protección de microrefugios y continuación de sus actividades en el sitio?

- 19. ¿cómo percibe estos factores para usted y otros campesinos/la montaña medio ambiente/la comunidad?

- 20. ¿Qué papel cree que los campesinos pueden y deben jugar en la planificación de microrefugios para la conservación?

- 21. ¿Qué papel cree que los campesinos pueden y deberían desempeñar en el proceso de declaración de impacto ambiental?

- 22. ¿Se prevé una actividad de ecoturismo posible hacia el sitio?

¿Alternativamente, se prevé una actividad de Agroturismo posible hacia la granja donde vive o actividad de etnoturismo hacia la comunidad de montaña

- 23. ¿Ha sido capaz de experimentar un cambio en las condiciones del clima? ¿Cree que las especies han respondido a estos cambios? Si es así, ¿cómo se registra esta respuesta?

- 24. ¿En caso de peligro de destrucción de estos bosques, en qué cree que las especies endémicas serán capaces de sobrevivir al cambio climático?

- 25. En su memoria, ¿recuerda una situación similar en las últimas décadas? Si es así, ¿cuál cree que es la razón de la repetición de la situación? 